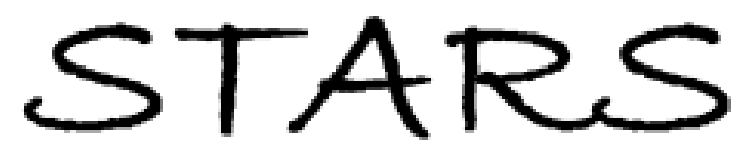

University of Central Florida

STARS

Faculty Bibliography 2010s

Faculty Bibliography

$1-1-2011$

\title{
Contributions to anelasticity in langasite and langatate
}

Ward L. Johnson

Sudook A. Kim

Satoshi Uda

Christine F. Rivenbark

University of Central Florida

Find similar works at: https://stars.library.ucf.edu/facultybib2010

University of Central Florida Libraries http://library.ucf.edu

This Article is brought to you for free and open access by the Faculty Bibliography at STARS. It has been accepted for inclusion in Faculty Bibliography 2010 s by an authorized administrator of STARS. For more information, please contactSTARS@ucf.edu.

\section{Recommended Citation}

Johnson, Ward L.; Kim, Sudook A.; Uda, Satoshi; and Rivenbark, Christine F., "Contributions to anelasticity in langasite and langatate" (2011). Faculty Bibliography 2010s. 1442.

https://stars.library.ucf.edu/facultybib2010/1442

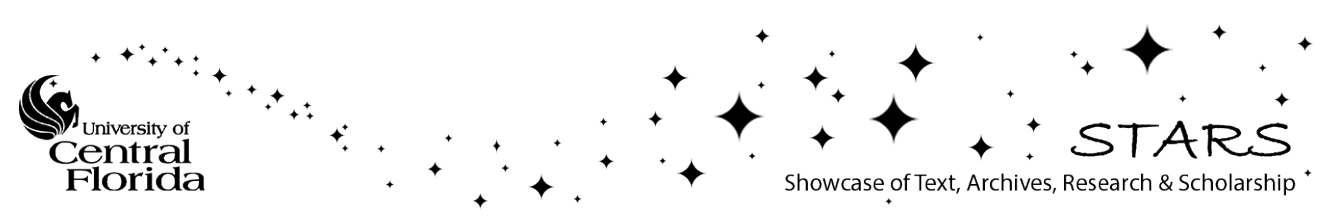




\section{Contributions to anelasticity in langasite and langatate}

Cite as: J. Appl. Phys. 110, 123528 (2011); https://doi.org/10.1063/1.3672443

Submitted: 20 July 2011 . Accepted: 27 November 2011 . Published Online: 30 December 2011

Ward L. Johnson, Sudook A. Kim, Satoshi Uda, and Christine F. Rivenbark

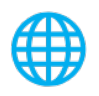

View Online

\section{ARTICLES YOU MAY BE INTERESTED IN}

Characterization of high temperature piezoelectric crystals with an ordered langasite structure

Journal of Applied Physics 105, 114107 (2009); https://doi.org/10.1063/1.3142429

Elastic Wave Propagation in Piezoelectric Semiconductors

Journal of Applied Physics 33, 40 (1962); https://doi.org/10.1063/1.1728525

The effective electromechanical coupling coefficient of piezoelectric thin-film resonators Applied Physics Letters 86, 022904 (2005); https://doi.org/10.1063/1.1850615

\section{Applied Physics Reviews} Now accepting original research

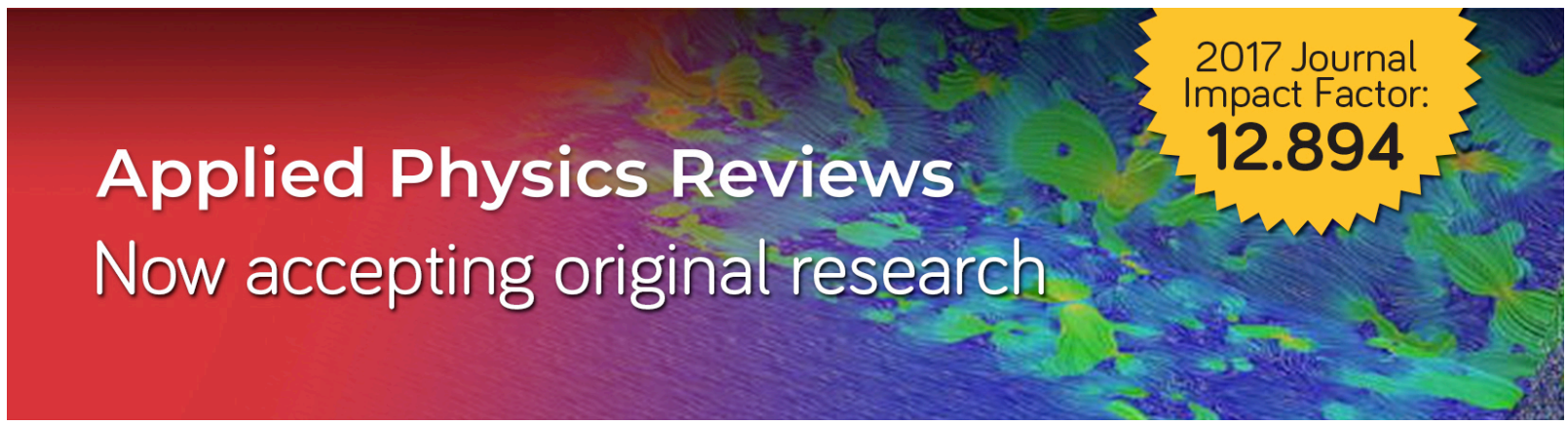




\title{
Contributions to anelasticity in langasite and langatate ${ }^{a)}$
}

\author{
Ward L. Johnson, ${ }^{1, b)}$ Sudook A. Kim, ${ }^{1}$ Satoshi Uda, ${ }^{2}$ and Christine F. Rivenbark ${ }^{3, c)}$ \\ ${ }^{1}$ National Institute of Standards and Technology, 325 Broadway, Boulder, Colorado 80305, USA \\ ${ }^{2}$ Institute for Materials Research, Tohoku University, 2-1-1 Katahira, Aoba-ku, Sendai 980-8577, Japan \\ ${ }^{3}$ Advanced Materials Processing and Analysis Center (AMPAC), University of Central Florida, Orlando, \\ Florida 32816, USA
}

(Received 20 July 2011; accepted 27 November 2011; published online 30 December 2011)

\begin{abstract}
Maximization of the quality factors $Q$ of langasite (LGS) and langatate (LGT) is necessary for optimal performance of acoustic resonators of these materials in frequency-control and hightemperature sensing applications. In this report, measurements and least-squares analysis of $Q^{-1}$ as a function of ultrasonic frequency and temperature of undoped LGS (100 K to $750 \mathrm{~K}$ ) and LGT (300 K to $760 \mathrm{~K}$ ) reveal a superposition of physical effects, including point-defect relaxations and intrinsic phonon-phonon loss. In LGS, these effects are superimposed on a large temperature-dependent background with weak frequency dependence that is interpreted as arising from a relaxation process with a distribution of activation energies. This distributed relaxation is suggested to be a result of anelastic kink migration. No evidence for a significant background of this form is found in the LGT specimen, consistent with the lower measured dislocation etch-pit density of this crystal. The analysis of the dependence of $Q^{-1}$ of LGT on frequency and temperature indicates that, at near-ambient temperatures, the damping in this specimen is close to the intrinsic limit determined by phononphonon interactions. Piezoelectric/carrier relaxation, which must occur at sufficiently elevated temperatures, is found not to be a significant contribution to $Q^{-1}$, relative to defect-related contributions, in either LGS or LGT in the measured range of temperatures. (C) 2011 American Institute of Physics. [doi:10.1063/1.3672443]
\end{abstract}

\section{INTRODUCTION}

The physical properties of langasite (LGS, $\left.\mathrm{La}_{3} \mathrm{Ga}_{5} \mathrm{SiO}_{14}\right)$, langatate (LGT, $\mathrm{La}_{3} \mathrm{Ga}_{5.5} \mathrm{Ta}_{0.5} \mathrm{O}_{14}$ ), and similar disordered and ordered piezoelectric crystals in the P321 crystal class (32-point group) have become a subject of increasing research over the past two decades because of their application in resonant acoustic devices for sensing and frequency control. $^{1,2}$ These compounds have a number of properties that make them attractive, relative to more traditional piezoelectric and ferroelectric materials, including their relatively high piezoelectric coefficients (two to three times greater than that of quartz ${ }^{3,4}$ ), relatively low stress coefficients, ${ }^{5-8}$ and ability to be grown by the Czochralski, vertical Bridgman, or float-zone methods. The fact that they have no phase transitions below their melting temperatures $(>1700 \mathrm{~K})$ makes them a particularly compelling choice for hightemperature sensing applications, and this has motivated much of the research on these materials in recent years. In comparison, quartz undergoes a structural phase transition at $846 \mathrm{~K}$, and perovskite ferroelectrics, such as lithium niobate and lithium tetraborate, decompose or undergo ferroelectricto-paraelectric transitions in the temperature range of $\sim 550$ $\mathrm{K}$ to $\sim 750 \mathrm{~K}$, leading to a loss of electromechanical coupling. The material currently most competitive with

\footnotetext{
a) This manuscript is a contribution of the National Institute of Standards and Technology and is not subject to copyright in the United States.

b) Author to whom correspondence should be addressed. Electronic mail: wjohnson@boulder.nist.gov.

${ }^{c}$ Current address: Krystal Engineering LLC, 1429 Chaffee Dr., Titusville, Florida 32780, USA.
}

LGS-type materials for high-temperature resonator applications is gallium orthophosphate $\left(\mathrm{GaPO}_{4}\right)$, which has been successfully operated at temperatures approaching the phase transition at $\sim 1200 \mathrm{~K}$ and has been reported to have relatively low damping at elevated temperatures. 9 However, obstacles to wide-spread application of $\mathrm{GaPO}_{4}$ resonators include its relatively high cost and limited availability, which are associated with the difficult and time-intensive growth process.

To achieve optimal resolution in sensing applications and high accuracy and stability in frequency-control applications, the quality factor $Q$ of a crystal must be maximized, because a lower $Q$ leads to increased noise and associated uncertainty in the resonant frequency of an oscillator. Previous resonant ultrasonic and electrical measurements of LGS and LGT have revealed several peaks in $Q^{-1}$ as a function of temperature. ${ }^{2,9-15}$ At cryogenic and near ambient temperatures, LGS and LGT are reported to have similar relaxation peaks, with maxima of $Q^{-1}$ occurring near $273 \mathrm{~K}$ and $233 \mathrm{~K}$, respectively, at $10 \mathrm{MHz}{ }^{10,11}$ Measurements directly comparing high-temperature $Q^{-1}$ of these two materials are limited. ${ }^{11}$ The peaks in damping of LGS and LGT are reported to be superimposed on a background that increases monotonically with temperature. ${ }^{2,9-15}$

The physical mechanisms responsible for the peaks and temperature-dependent background in LGS and LGT have not been determined, with the exception that Fritze, Schulz, and coworkers ${ }^{2,13}$ have found the highest-temperature peak in LGS to arise from the movement of charge carriers in the oscillating piezoelectric field, with a relaxation time equal to the inverse of the dielectric relaxation frequency. In this 
paper, we present analysis and additional measurements of the contributions to $Q^{-1}$ in LGS and LGT, with the aim of providing further insight into physical mechanisms that dominate the acoustic loss at various temperatures.

\section{SPECIMENS}

An LGS specimen was fabricated from a crystal grown by Mitsubishi Materials Corporation with the Czochralski method. ${ }^{65}$ The crystal boule had a diameter of $100 \mathrm{~mm}$ and was pulled along the $<01 \overline{1} 1>$ axis from an iridium crucible with a diameter of 160-170 $\mathrm{mm}$. The growth atmosphere was argon with 2-4 vol. \% oxygen. This level of oxygen was employed to provide a reasonable balance between the beneficial suppression of evaporation of gallium and the detrimental oxidation of the iridium crucible. The homogeneity of elastic properties of similarly grown crystals was evaluated through measurements of the distribution of surfaceacoustic-wave velocities of wafers. These velocities had a standard deviation of $\sim 100 \mathrm{ppm}$. A piece of this crystal was fabricated into an unplated, plano-convex, Y-cut disk with a diameter of $14 \mathrm{~mm}$. The spherical surface had a radius of $265 \mathrm{~mm}$ (2 diopters). The thickness at the center was 0.68 $\mathrm{mm}$, which corresponds to a frequency of $2.0 \mathrm{MHz}$ for the fundamental thickness-shear trapped resonant mode. The methods of cutting, lapping, polishing, and cleaning the specimen are described by Smythe. ${ }^{16}$ Final cleaning was performed with acetone followed by ethanol.

The LGS crystal has a light orange tint, similar to that reported in other studies of LGS, LGT, and crystallographically similar piezoelectric materials grown in partial oxygen atmospheres. ${ }^{17-21}$ The point defects responsible for such coloration in this family of materials have not been determined but have been shown, in several studies, to be related to oxygen content. $^{17-21}$

A lower-loss "state of the art" LGT resonator was also fabricated to provide a comparison of its anelastic properties with those of langasite. This colorless crystal was grown at the Advanced Materials Processing and Analysis Center of the University of Central Florida by use of the Czochralski method with an iridium crucible. The atmosphere during growth was pure nitrogen. Nominal dimensional parameters and methods of fabricating a plano-convex Y-cut disk from this crystal were the same as those employed for the LGS specimen.

To enable estimates of dislocation density, the crystals were etched, after ultrasonic measurements, in orthophosphoric acid at $130{ }^{\circ} \mathrm{C}$ for $1 \mathrm{~h}$. Dislocation etch pits of the LGT were determined to have a density of $3 \times 10^{3} / \mathrm{cm}^{2}$, and those of LGS were determined to have a density one to two orders magnitude greater than that of LGT, with substantial inhomogeneity of etch-pit density and the presence of other etch structures and surface flaws introducing uncertainty into analysis of the images.

The crystals had no electrodes deposited on the surfaces to avoid potential contributions to $Q^{-1}$. Although the damping effects of electrodes are expected to be insignificant at ambient temperatures, $Q^{-1}$ of typical metals increases greatly at elevated temperatures, ${ }^{22,23}$ so that this contribution to the resonator $Q^{-1}$ could potentially become significant at the highest temperatures employed in this study, even with electrode thicknesses on the order of a hundred nanometers.

\section{EXPERIMENTAL TECHNIQUE}

As depicted in Fig. 1, each specimen was supported near its edge on three sapphire spheres with diameters of $2.4 \mathrm{~mm}$ and was constrained horizontally by three alumina spheres with diameters of $6.4 \mathrm{~mm}$. This configuration provides mechanical support, with minimal contact area, for the specimen in regions where the amplitude of the trapped-mode vibrations is small, so that negligible damping is introduced. Two noncontacting copper electrodes with diameters of 7.7 $\mathrm{mm}$ and a spacing of $2.7 \mathrm{~mm}$ were supported by boron nitride fixtures (not shown) above and below the specimen to facilitate direct noncontacting piezoelectric transduction. The specimen, electrodes, and supporting structure (Fig. 1) were inside a copper chamber with a diameter of $6.2 \mathrm{~cm}$. This chamber was placed inside a larger chamber that included radiative shielding, resistive heating wires, and water-cooling tubes. All of these components were contained within a turbo-pumped bell jar.

For LGS measurements below ambient temperatures, cooling was accomplished by passing liquid nitrogen through the tubes that were designed for water cooling of the furnace shielding at elevated temperatures. Temperatures were measured with a thermocouple attached to the inner chamber. To minimize thermal gradients, no active heating or cooling was employed during the measurements, except in the temperature range of $275 \mathrm{~K}$ to $300 \mathrm{~K}$. Instead, temperature ramps were implemented through passive thermal exchange during heating from $100 \mathrm{~K}$ to $275 \mathrm{~K}$ and during cooling from $750 \mathrm{~K}$ to $300 \mathrm{~K}$. During passive heating at cryogenic temperatures, the ramp rate was in the range of $0.001 \mathrm{~K} / \mathrm{s}$ to $0.005 \mathrm{~K} / \mathrm{s}$ (decreasing with increasing temperature). During passive cooling from elevated temperatures, the ramp rate was initially $0.02 \mathrm{~K} / \mathrm{s}$ at $750 \mathrm{~K}$ and decreased continuously on cooling toward ambient temperatures, reaching $0.005 \mathrm{~K} / \mathrm{s}$ at 500 $\mathrm{K}$ and $0.001 \mathrm{~K} / \mathrm{s}$ at $300 \mathrm{~K}$.

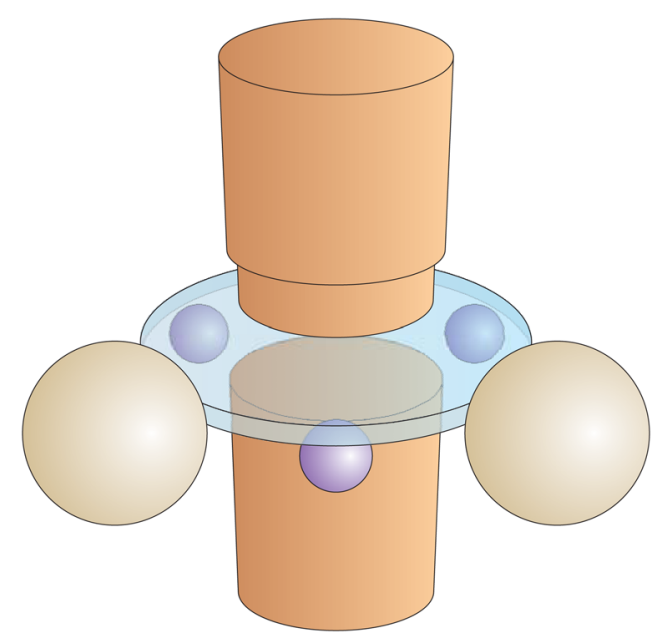

FIG. 1. (Color online) Disk-shaped specimen supported near its edges by three sapphire spheres and restrained horizontally by three larger alumina spheres. Noncontacting copper electrodes are above and below the specimen. 
The LGS measurements were performed either under high vacuum or with a continuous flow of helium that first was passed through a titanium gettering furnace to remove oxygen. The pressure of helium was $1.3 \mathrm{~Pa}$ at temperatures below $300 \mathrm{~K}$ and $0.6 \mathrm{~Pa}$ to $2.7 \mathrm{~Pa}$ between $300 \mathrm{~K}$ and $393 \mathrm{~K}$. Above $393 \mathrm{~K}$, no helium was introduced, and vacuum pressures on the order of $1 \times 10^{-4} \mathrm{~Pa}$ were maintained.

LGT measurements were performed only above $300 \mathrm{~K}$. The sequence of vacuum and exchange gas and the resulting ramp rates during passive cooling were similar to those employed for LGS.

To excite a resonant vibrational mode of the specimen, a sinusoidal tone burst with a duration of $1 \mathrm{~ms}$ to $5 \mathrm{~ms}$ was applied to the electrodes at a frequency close to resonance. Freely decaying vibrations of the specimen following excitation were detected through voltages induced on the electrodes. $Q^{-1}$ was determined (in real time) from the rate of exponential decay of the amplitude calculated from the magnitude of the components of the signal that were in phase and out of phase with the reference-driving sinusoid. $^{22}$ In the LGS measurements, the resonant frequency was determined from the rate of change of phase of the decaying signal. ${ }^{22}$ In the LGT measurements, which were performed at a later date, the resonant frequency was determined from a complex FFT of the decaying in-phase and out-of-phase components of the signal; this algorithm was found to be more robust in the presence of signal noise and spurious resonances. Neither of these methods for determining the resonant frequency requires the driving frequency to be exactly at resonance. After each measurement, the driving frequency for each mode was automatically set to the anticipated frequency at the next measured temperature. This was estimated from the current frequency and current rate of change of frequency with temperature.

\section{MEASUREMENTS}

Figure 2 shows measurements of $Q^{-1}$ of LGS versus the inverse of temperature $T$ from $100 \mathrm{~K}$ to $740 \mathrm{~K}$ for the four lowest thickness-shear resonant frequencies $(2.03 \mathrm{MHz}, 6.05$ $\mathrm{MHz}, 9.96 \mathrm{MHz}$, and $13.94 \mathrm{MHz}$ at the lowest measured temperature). These measurements were partially reported previously by Johnson et al. ${ }^{11}$ Sharp peaks (extending over only a few degrees), which appear in some temperature ranges, have been deleted from the results presented in Fig. 2 to provide a more clear presentation of the relevant results. Such peaks are understood to arise from the phenomenon of "activity dips" associated with coupling to spurious vibrational modes with flexural displacement components and correspondingly poor vibrational trapping. ${ }^{24}$

Two clear frequency-dependent peaks are shown in Fig. 2, superimposed on a background that is only weakly dependent on temperature near $100 \mathrm{~K}$ and increases monotonically with temperature above $\sim 140 \mathrm{~K}$. This background is similar to that reported in resonant-ultrasound spectroscopic (RUS) measurements of peak widths, ${ }^{14}$ attenuation measurements of propagating surface-acoustic waves, ${ }^{25}$ and electri-
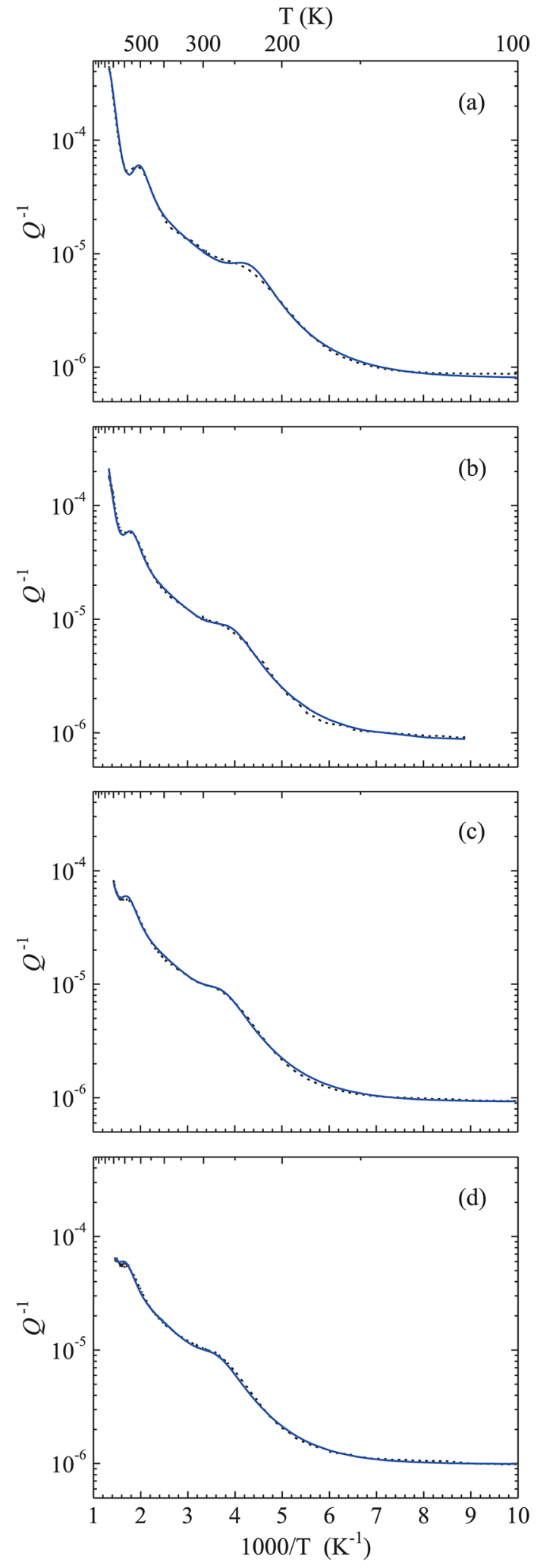

FIG. 2. (Color online) Measured $Q^{-1}$ of LGS (dashed lines) and multifrequency least-squares fit to function $Q_{2}^{-1}$ (Eq. (15)) (solid lines) at (a) 2.03 $\mathrm{MHz}$, (b) $6.05 \mathrm{MHz}$, (c) $9.96 \mathrm{MHz}$, and (d) $13.94 \mathrm{MHz}$.

cal measurements of effective mechanical viscosity based on equivalent-circuit models. ${ }^{2,13,26,27}$

Figure 3 shows measurements of $Q^{-1}$ of LGT at 6.02 $\mathrm{MHz}, 10.03 \mathrm{MHz}$, and $14.03 \mathrm{MHz}$ over a narrower temperature range ( $300 \mathrm{~K}$ to $760 \mathrm{~K}$ ). Measurements of the fundamental 
$2 \mathrm{MHz}$ mode of this specimen were not pursued as a function of temperature, because room temperature measurements showed this mode to have a $Q^{-1}$ that was an order of magnitude greater than that of the measured overtones, which indicated that vibrational trapping of this mode was poor. The form of the dependence of $Q^{-1}$ on temperature and frequency shown in Fig. 3 is qualitatively similar to that of LGS shown in Fig. 2, although the overall magnitude $Q^{-1}$ is more than an order of magnitude lower.

\section{ANALYSIS}

In this section, analytical expressions for $Q^{-1}$ are presented and fit to the data of Figs. 2 and 3 to provide a
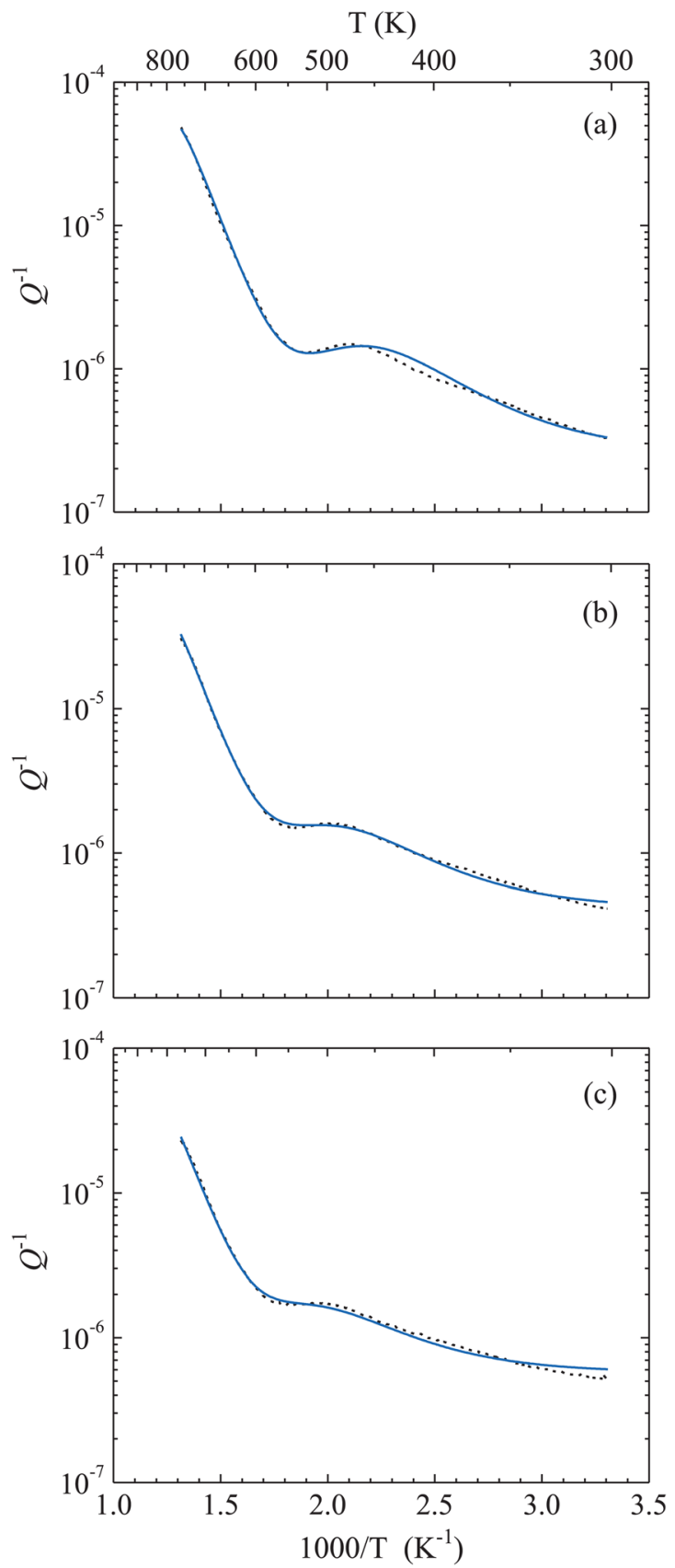

FIG. 3. (Color online) Measured $Q^{-1}$ of LGT (dashed lines) and multifrequency least-squares fit to function $Q_{3}^{-1}$ (Eq. (19)) (solid lines) at (a) 6.02 $\mathrm{MHz}$, (b) $10.03 \mathrm{MHz}$, and (c) $14.03 \mathrm{MHz}$. basis for physical interpretation of these data. First, functional forms for the damping arising from phonon-phonon interactions, point-defect relaxations, and piezoelectric/ carrier relaxation are considered. Expressions for these contributions are combined with an initial empirical expression for the temperature-dependent background and fit through least-squares analysis to the data. Some of the parameters obtained from these initial fits are found to be implausible, based on previous theory and measurements. A second least-squares analysis of these data is then performed with analytical expressions that are more physically credible.

Phonon-phonon interactions lead to an intrinsic contribution to $Q^{-1}$ that is present even in perfect crystals. ${ }^{28}$ This contribution is proportional to frequency $f$ in the megahertz range $^{29}$ and is responsible for $Q f$ being approximately constant in high-quality piezoelectric resonators at room temperature. In typical materials, phonon-phonon loss is not strongly dependent on temperature above $100 \mathrm{~K}$ (see, for example, results for quartz, silicon, and germanium summarized by Mason $^{29}$ ). Therefore, the fact that $Q^{-1}$ in LGS increases with $f$, but is weakly dependent on temperature in the range of $1000 / T>\sim 7 \mathrm{~K}^{-1}$ is consistent with phononphonon loss being a significant fraction of $Q^{-1}$ at these low temperatures.

The two peaks that are visible in Fig. 2 and the single peak in Fig. 3 appear to be consistent with anelastic point-defect relaxations with respect to the general form of their dependence on temperature and frequency. Pointdefect relaxations have the form of a Debye function with inverse temperature dependence of the relaxation strength, ${ }^{30}$

$$
Q^{-1}=\frac{\Delta}{T} \frac{\omega \tau}{1+\omega^{2} \tau^{2}}
$$

where $\tau$ is an Arrhenius function of temperature,

$$
\tau=\gamma \exp (U / k T)
$$

$\Delta$ is a temperature-independent factor in the relaxation strength, $\omega$ is the acoustic angular frequency, $U$ is an activation energy, and $\gamma$ is a time constant corresponding to the inverse of the attempt rate of thermally activated hopping of point-defects over potential barriers. ${ }^{30}$

In the range of $1000 / T$ between approximately $2 \mathrm{~K}^{-1}$ and $5 \mathrm{~K}^{-1}$, the background of $Q^{-1}$ in Fig. 2 is roughly linear on the semilog scale. However, at temperatures above the maximum of the second peak (at $2 \mathrm{MHz}, 1000 / T<1.7$ $\mathrm{K}^{-1}$ ), the dependence of $Q^{-1}$ on temperature is stronger. This suggests the presence of an additional relaxation peak with a maximum above the measured temperature range.

A peak with a maximum at a temperature above the measured range of Figs. 2 and 3 is also expected from theories of wave propagation in piezoelectric crystals. According to Hutson and White, ${ }^{31}$ coupled acoustic and electric fields in piezoelectric crystals with finite conductivity produce time-dependent currents and associated space charge and strain with components that are out of phase with the 
acoustic stress, resulting in a piezoelectric/carrier contribution to $Q^{-1}$ that is given by

$$
\begin{aligned}
Q_{c}^{-1}(\omega, T) & =\Delta_{c} \frac{\omega_{c} / \omega}{1+2\left(\omega_{c} / \omega_{D}\right)+\left(\omega / \omega_{D}\right)^{2}+\left(\omega_{c} / \omega\right)^{2}} \\
& =\Delta_{c} \frac{\omega / \omega_{c}}{1+\left(\omega / \omega_{c}\right)^{2}+2 \zeta+\zeta^{2}},
\end{aligned}
$$

where

$$
\begin{gathered}
\Delta_{c} \equiv K^{2} \equiv \frac{e_{i j k}^{2}}{C_{l m n p}^{E} \epsilon_{r S}^{S}}, \\
\zeta \equiv \frac{\omega^{2}}{\omega_{c} \omega_{D}} .
\end{gathered}
$$

In this equation, $K$ is the electromechanical coupling coefficient, $C_{\text {lmn }}^{E}$ is the elastic stiffness at constant electric field, $e_{i j k}$ is the piezoelectric coefficient, and $\epsilon_{r s}^{S}$ is the dielectric permittivity at constant strain. The dielectric relaxation frequency $\omega_{c}$ is given by

$$
\omega_{c}=\sigma / \epsilon_{r s}^{S},
$$

where $\sigma$ is the conductivity. The "diffusion frequency" $\omega_{\mathrm{D}}$ is given by

$$
\omega_{D}=\frac{q C_{\operatorname{lmn}}^{E}}{k T p \mu \rho},
$$

where $q$ is the charge of individual carriers, $\mu$ is the carrier mobility, $\rho$ is the material density, $k$ is Boltzmann's constant, and $p$ is the fraction of acoustically produced space charge that is mobile. $C_{\text {lmnp }}^{E}, e_{i j k}, \epsilon_{r s}^{S}, \omega_{c}, \omega_{D}, \sigma$, and $\mu$ are all dependent on temperature.

The relevant subscripted indices for $C_{l m n p}^{E}, e_{i j k}$, and $\epsilon_{r s}^{S}$ in Eqs. (4)-(7) are those that enter into the equation of motion for the particular waves and crystal symmetries of interest. For a through-thickness shear mode in a Y-cut P321 crystal, the relevant coefficients are $e_{11}, C_{66}^{E}$, and $\epsilon_{1}^{S}$ (where reduced indices are employed). ${ }^{32,33}$

An estimate of the maximum $\zeta$ during the measurements can be obtained by inserting into Eqs. (5)-(7) values of $C_{66}^{E}, \epsilon_{1}^{S}$, and $\rho$ summarized by Kosinski et al., ${ }^{4}$ along with values for temperature-dependent $\sigma$ and $\mu$ in undoped LGS reported by Seh and Tuller. ${ }^{34}$ At the highest measured temperature of $740 \mathrm{~K}$, this leads to $\zeta \approx 2 p \times 10^{-9}$ (where $p<1)$ at $14 \mathrm{MHz}$, assuming $|q|$ is equal to two times the electronic charge (as expected for oxygen vacancies being the dominant charge carriers in this material ${ }^{34}$ ). Since $\zeta$ is much less than 1, the dependence of Eq. (3) on carrier diffusion is negligible and $Q_{c}^{-1}$ assumes a simple Debye form,

$$
Q_{c}^{-1}(\omega, T)=\Delta_{c} \frac{\omega \tau_{c}}{1+\omega^{2} \tau_{c}^{2}},
$$

where $\tau_{c} \equiv 1 / \omega_{c}$.
Following Seh and Tuller, ${ }^{34} \sigma$ in nominally undoped or acceptor-doped LGS is assumed to have the form

$$
\sigma=\frac{c_{0}}{T} \exp \left(-U_{c} / k T\right)
$$

where $U_{c}$ is an activation energy. Therefore, the dielectric relaxation time is given by

$$
\tau_{c}=\frac{\epsilon_{1}^{S} T}{c_{0}} \exp \left(U_{c} / k T\right) .
$$

Considering the various anticipated contributions to $Q^{-1}$ described above, the function form of $Q^{-1}$ may be taken, for the purpose of initial fitting of the data, as

$$
\begin{aligned}
Q_{1}^{-1}= & \sum_{i=1}^{2} \frac{\Delta_{i}}{T} \frac{\omega \tau_{i}}{1+\omega^{2} \tau_{i}^{2}}+\frac{\delta_{c}}{\epsilon_{1}^{S}} \frac{\omega \tau_{c}}{1+\omega^{2} \tau_{c}^{2}} \\
& +\Delta^{\prime} \omega^{n} \exp \left(-U^{\prime} / k T\right)+B \omega+C,
\end{aligned}
$$

where

$$
\begin{gathered}
\tau_{i}=\gamma_{i} \exp \left(U_{i} / k T\right), \\
\delta_{c}=e_{11}^{2} / C_{66}^{E} .
\end{gathered}
$$

The term $B \omega$ (with constant $B$ ) is intended to approximate the phonon-phonon loss. The approximation of temperature independence of this contribution is considered to be reasonable in light of the above-mentioned weak temperature dependence that is reported ${ }^{29}$ for the phonon-phonon loss above $\sim 100 \mathrm{~K}$ in other materials and the apparent increasing dominance of other contributions to $Q^{-1}$ above $\sim 200 \mathrm{~K}$ in Figs. 2 and 3. The empirical temperature-dependent background term allows for frequency dependence of the form $\omega^{n}$. The constant $C$ is an empirical term that is intended to partially enable inclusion of any contributions to $Q^{-1}$ that are not internal material losses, such as that arising from energy loss to the structure supporting the specimen.

Least-squares analysis was performed on the logarithms of $Q_{1}^{-1}$ and the measured $Q^{-1}$ of LGS to achieve comparable weighting of points (since the values of $Q^{-1}$ in Fig. 2 extend over more than two orders of magnitude). In this analysis, each of the frequencies of the four modes was approximated as constant, with the values taken as those measured at the lowest temperature. Actual frequencies varied less than $0.7 \%$ over the measured temperature range, which is expected to introduce insignificant uncertainty in the determination of the frequency dependence of $Q^{-1}$. Corresponding to this approximation, the temperature dependence of $C_{66}^{E}$ is not included in the expression for $\delta_{c}$ (Eq. (13)). Temperature dependence of $e_{11}$ is also neglected in the least-squares analysis, since temperature coefficients of $e_{11}$ are not readily available. Therefore, $\delta_{c}$ is approximated as having no temperature dependence. The temperature-dependent dielectric constant of LGS is taken to be that reported by Schreuer et al. ${ }^{14}$

Simultaneous fitting of the LGS data at the four frequencies yields the following parameters for the function $Q_{1}^{-1}$ : 


$$
\begin{aligned}
\Delta_{1} & =1.96 \times 10^{-3}, \\
\gamma_{1} & =1.35 \times 10^{-12} \mathrm{~s}, \\
U_{1} & =0.224 \mathrm{eV}, \\
\Delta_{2} & =2.25 \times 10^{-2} \\
\gamma_{2} & =1.45 \times 10^{-14} \mathrm{~s}, \\
U_{2} & =0.676 \mathrm{eV}, \\
\delta_{c} & =1.66 \times 10^{-13} \mathrm{Ss} / \mathrm{m}, \\
c_{0} & =2.47 \times 10^{7} \mathrm{SK} / \mathrm{m}, \\
U_{c} & =1.10 \mathrm{eV} \\
\Delta^{\prime} & =3.56 \times 10^{-3}, \\
U^{\prime} & =0.091 \mathrm{eV} \\
n & =-0.15 \\
B & =1.59 \times 10^{-15} \mathrm{~s}, \\
C & =8.05 \times 10^{-7} .
\end{aligned}
$$

This fit (not shown) closely approximates the measured $Q^{-1}$ at all four frequencies over the entire temperature range, having a standard deviation of 0.019 of the measured $\log _{10} Q^{-1}$ relative to the function $\log _{10} Q_{1}^{-1}$.

The values obtained for $\Delta_{1}, \Delta_{2}, \gamma_{1}, \gamma_{2}, U_{1}$, and $U_{2}$ of the first two Debye functions are typical of point-defect relaxations. ${ }^{30}$ Therefore, the fit supports our hypothesis that point defects are the physical source of these peaks.

The parameters obtained for the piezoelectric/carrier contribution are much less convincing, with the exception of $U_{c}$. Published values of activation energy of $\sigma T$ or $\sigma$ in LGS range from $\sim 0.88 \mathrm{eV}$ to $\sim 1.1 \mathrm{eV}$ for single crystals ${ }^{27,35,36}$ or sintered powders. ${ }^{34}$ Domoroshchina et al. ${ }^{36}$ found substantial variation in activation energy within single crystals grown by the Czochralski method. Considering this range of reported activation energies, our fit value of $1.10 \mathrm{eV}$ for $U_{c}$ appears to be consistent with piezoelectric/carrier relaxation. However, the value obtained for $\delta_{c}$ is not consistent with expectations from Eq. (13), based on published values of $e_{11}$ and $C_{66}^{E}$. With $e_{11}=-0.52 \mathrm{C} / \mathrm{m}^{2}$ and $C_{66}^{E}=41 \mathrm{GPa}$ at 750 $\mathrm{K}$ in LGS, ${ }^{2,37} \mathrm{Eq}$. (13) yields a value of $6.6 \times 10^{-12}$ for $\delta_{c}$, which is 40 times greater than that obtained from the leastsquares fit to function $Q_{1}^{-1}$ (Eq. (18)). The fit value of $c_{0}$ obtained for LGS is also much different from expectations based on the theory of Hutson and White ${ }^{31}$ and previous electrical measurements of the parameters in Eq. (9). From the results of Seh and Tuller, ${ }^{34} c_{0}$ in LGS is expected to be approximately $5 \times 10^{4} \mathrm{SK} / \mathrm{m}$ in undoped LGS, which is three orders of magnitude smaller than the value obtained from the fit of Eq. (14).

A similar discrepancy occurs with the fitting of the LGT data of Fig. 3 to function $Q_{1}^{-1}$ (with use of the temperature coefficients of $\epsilon_{1}^{S}$ reported by Schreuer et al. ${ }^{14}$ ), which yields values of $0.95 \mathrm{eV}$ for $U_{c}, 2.18 \times 10^{-14} \mathrm{Ss} / \mathrm{m}$ for $\delta_{c}$, and $5.63 \times 10^{6} \mathrm{SK} / \mathrm{m}$ for $c_{0}$. Published values of activation energies of $\sigma T$ are in the range of $0.80 \mathrm{eV}$ to $1.02 \mathrm{eV}$ at temperatures below $1000 \mathrm{~K},{ }^{20,38}$ consistent with our value for $U_{c}$. However, the fit value of $\delta_{c}$ is different from that predicted by Eq. (4), based on previously published measurements.
Dankov et al. ${ }^{39}$ reported a value of $\sim 0.095$ at $750 \mathrm{~K}$ for the electromechanical coupling coefficient $K$. Based on this and a value of $\epsilon_{1}=22.4 \epsilon_{0}$ at $750 \mathrm{~K}$ reported by Schreuer et al., ${ }^{14}$ Eqs. (4) and (13) lead to an estimate of $1.8 \times 10^{-12} \mathrm{Ss} / \mathrm{m}$ for $\delta_{c}$, which is $\sim 83$ times greater than the value of $\delta_{c}$ obtained from fitting the LGT data to function $Q_{1}^{-1}$.

We conclude that our initial hypothesis that piezoelectric/carrier relaxation dominates $Q^{-1}$ at our highest measured temperatures is incorrect. As an alternate explanation for the more rapid rise in $Q^{-1}$ at these temperatures, we suggest the presence of a third point-defect relaxation with an activation energy close to that of the piezoelectric/carrier relaxation, but with a substantially different $\gamma$. This alternate hypothesis is supported by previously published electrical measurements on undoped LGS by Fritze and coworkers, ${ }^{2}$ which show evidence for an unidentified relaxation peak near $930 \mathrm{~K}$ at $5 \mathrm{MHz}$ and a piezoelectric/carrier peak appearing at a higher temperature of $\sim 1180 \mathrm{~K}$. The identification of the piezoelectric/carrier peak in this work is consistent with an estimate of $1200 \mathrm{~K}$ for the peak temperature based on Eqs. (8)-(10) and published values ${ }^{14,34}$ of $\sigma(T)$ and $\epsilon_{1}^{s}(T)$.

The results listed in Eq. (14) for the fit parameters of the temperature-dependent background term are also problematic. In particular, the small value obtained for $n$, combined with the exponential dependence on $1 / T$, appears to be inconsistent with a single relaxation effect. A singlerelaxation Debye function has an approximate inverse temperature dependence only in the limit of high $\omega$, and, in this case, the value of $n$ is -1 . We note that relatively weak frequency dependence of $Q^{-1}$ occurs in materials with anelastic relaxations that have distributions of relaxation times, such as those arising from anelastic dislocation motion. Considering this, we proceed with a superposition of Debye functions as the functional form of the temperature-dependent background and assume, for simplicity, a continuous log-normal distribution of relaxation strengths as a function of activation energy, which satisfies the requirement of zero relaxation strength at zero activation energy. The validity of this function and possible alternate functions based on various published physical models are discussed further in Sec. VI.

The revised form of $Q^{-1}$, with this distributed-relaxation function for the background and an additional point-defect Debye function replacing the piezoelectric/carrier relaxation, becomes

$$
\begin{aligned}
Q_{2}^{-1}= & \sum_{i=1}^{3} \frac{\Delta_{i}}{T} \frac{\omega \tau_{i}}{1+\omega^{2} \tau_{i}^{2}}+B \omega+C+\Delta_{b} \int_{0}^{\infty} \frac{1}{u|\ln W|(2 \pi)^{1 / 2}} \\
& \times \exp \left[\frac{-\left(\ln u-\ln U_{b}\right)^{2}}{2(\ln W)^{2}}\right] \frac{\omega \tau_{b}}{1+\omega^{2} \tau_{b}^{2}} d u
\end{aligned}
$$

where

$$
\begin{aligned}
\tau_{i} & =\gamma_{i} \exp \left(U_{i} / k T\right), \\
\tau_{b} & =\gamma_{b} \exp (u / k T) .
\end{aligned}
$$

Figure 2 shows the results of fitting this function to the LGS data. The parameters obtained from the fit are 


$$
\begin{aligned}
\Delta_{1} & =1.72 \times 10^{-3}, \\
\gamma_{1} & =5.38 \times 10^{-13} \mathrm{~s}, \\
U_{1} & =0.240 \mathrm{eV}, \\
\Delta_{2} & =3.70 \times 10^{-2}, \\
\gamma_{2} & =3.72 \times 10^{-13} \mathrm{~s}, \\
U_{2} & =0.538 \mathrm{eV}, \\
\Delta_{3} & =0.667, \\
\gamma_{3} & =1.81 \times 10^{-13} \mathrm{~s}, \\
U_{3} & =0.866 \mathrm{eV}, \\
\Delta_{b} & =2.38 \times 10^{-4}, \\
\gamma_{b} & =7.03 \times 10^{-15} \mathrm{~s}, \\
U_{b} & =0.726 \mathrm{eV}, \\
W & =0.657, \\
B & =2.48 \times 10^{-15} \mathrm{~s}, \\
C & =7.70 \times 10^{-7} .
\end{aligned}
$$

As with the fit to function $Q_{1}^{-1}$ (Eq. (11)), this fit closely approximates the data at all the measured temperatures and frequencies, with a standard deviation of 0.019 in $\log _{10} Q^{-1}$.

Figure 4 shows the separate contributions to $Q^{-1}$ of LGS obtained from the fit of Eq. (18). The temperaturedependent background is the dominant contribution between $200 \mathrm{~K}$ and $500 \mathrm{~K}$. Above $500 \mathrm{~K}$, the second Debye peak rises above the background, and then, at the highest measured temperatures, the third Debye peak becomes dominant.

Fitting of the LGT data of Fig. 3 to the function $Q_{2}^{-1}$ leads to such a low value of $\Delta_{1}$ that the standard deviation of the fit is not significantly affected by deleting this lowest temperature relaxation from the fitting function. In other words, since the LGT data does not extend below ambient temperatures, the tail of the lowest relaxation peak, if present, is insignificant in the measured range of temperatures. The fit also provides little evidence for a temperaturedependent background of the form seen in LGS, the inte-

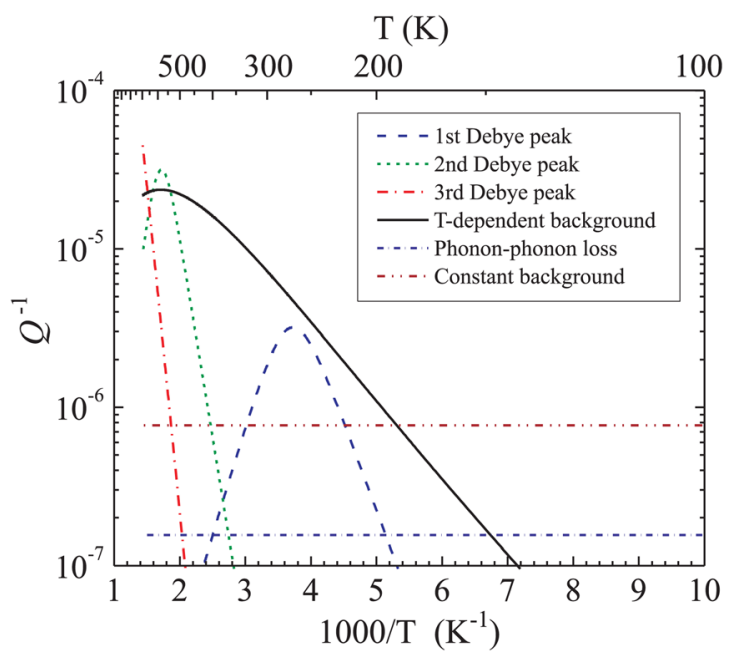

FIG. 4. (Color online) Separate contributions to $Q^{-1}$ of LGS at $10 \mathrm{MHz}$ obtained from fitting to the function $Q_{2}^{-1}$ at all four measured frequencies (Eq. (18)) grated term in $Q_{2}^{-1}$ playing a role of optimizing the fit at intermediate temperatures, with a maximum near that of the second relaxation. Therefore, after initial fitting of LGT data with function $Q_{2}^{-1}$, the fitting of the LGT data was repeated with the following greatly simplified function that does not include the lowest-temperature Debye peak or the temperature-dependent background,

$$
Q_{3}^{-1}=\sum_{i=2}^{3} \frac{\Delta_{i}}{T} \frac{\omega \tau_{i}}{1+\omega^{2} \tau_{i}^{2}}+B \omega+C .
$$

Parameters obtained from this fit are

$$
\begin{aligned}
\Delta_{2} & =1.08 \times 10^{-3}, \\
\gamma_{2} & =1.89 \times 10^{-11} \mathrm{~s}, \\
U_{2} & =0.292 \mathrm{eV}, \\
\Delta_{3} & =9.18 \times 10^{-2}, \\
\gamma_{3} & =1.31 \times 10^{-13} \mathrm{~s}, \\
U_{3} & =0.849 \mathrm{eV}, \\
B & =6.21 \times 10^{-15} \mathrm{~s}, \\
C & =2.92 \times 10^{-8},
\end{aligned}
$$

with a standard deviation of 0.024 in $\log _{10} Q^{-1}$. This function is plotted in Fig. 3. Corresponding to the lower $Q^{-1}$ of this LGT specimen over the entire measured temperature range, the values of $\Delta_{2}, \Delta_{3}$, and $C$ are all substantially less than those obtained for the LGS specimen.

The data and fit in Fig. 3 show some indication of broadening of the lower-temperature relaxation beyond that of a single Debye function. It appears that the activation energy has been lowered in the minimization of the square deviations to more closely match the width to the peaks. However, this leads to some mismatch of the peak positions that is especially visible in the $6 \mathrm{MHz}$ plot. Therefore, the activation energy $U_{2}$ obtained from the fit, which is substantially lower than that obtained for the second relaxation in LGS, may not accurately represent the mean activation energy of this possibly distributed relaxation. We refrain from exploring the introduction of additional parameters in the fitting function to more accurately match the data.

Figure 5 shows the separate contributions to the loss in LGT at $10 \mathrm{MHz}$. Unlike LGS, the intrinsic phononphonon loss is the dominant contribution to $Q^{-1}$ at ambient temperatures. The lower of the two point-defect relaxations is dominant between $\sim 390 \mathrm{~K}$ and $\sim 575 \mathrm{~K}$, and the higher point-defect relaxation is dominant at temperatures above $\sim 575 \mathrm{~K}$.

Although no detailed uncertainty analysis is performed on the least-squares-fit results listed in Eqs. (18) and (20), it may be useful to offer a few comments about potential systematic errors in some of the fit parameters. As briefly indicated above and described further below, the greatest uncertainty in the LGS analysis is the physical mechanism and associated functional form of the temperature-dependent background. During the course of this study, a number of other empirical, physically based forms for this background were tried in fitting the LGS data, including several forms 


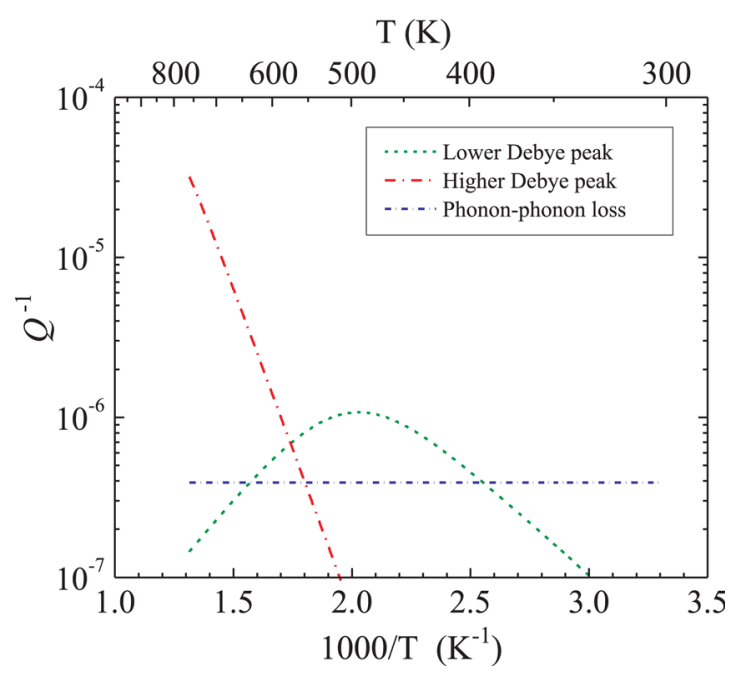

FIG. 5. (Color online) Separate contributions to $Q^{-1}$ of LGT at $10 \mathrm{MHz}$ obtained from fitting to the function $Q_{3}^{-1}$ at all three measured frequencies (Eq. (20)). The constant background term $C$ is below the plotted range at $2.9 \times 10^{-8}$.

taken from the literature on acoustic effects of dislocations (such as the dislocation string model ${ }^{40,41}$ with a Koehler ${ }^{40}$ distribution of dislocation loop lengths). These other functional forms for the background yielded either poor fits to the data or physically implausible parameters. However, for those functions that fit the data well (with standard deviations less than 0.025 in $\log _{10} Q^{-1}$ ), parameters obtained for the other contributions (the Debye peaks and phonon-phonon loss) were not greatly different from those listed in Eq. (18). In particular, values of $U_{1}$ obtained from these various fits were within $\sim 0.02 \mathrm{eV}$, values of $U_{2}$ were within $\sim 0.14 \mathrm{eV}$, and values of the phonon-phonon parameter $B$ were within a factor of $\sim 1.6$ of the values listed in Eq. (18). The fit parameters of Eq. (14) that were determined through the use of the initial empirical background function (Eq. (14)) provide an example of such differences. Parameters obtained for $Q^{-1}$ of LGT (Eq. (20)) have smaller uncertainties associated with the temperature-dependent background function, because this background, if present, is much smaller than that of LGS. However, as described above, there is substantial uncertainty in the value of $U_{2}$ associated with inaccuracy of the simple Debye form in fitting the second relaxation peak of LGT.

\section{DISCUSSION}

\section{A. Intrinsic phonon-phonon loss}

The value obtained for the intrinsic phonon-phonon loss coefficient $B$ of LGT corresponds to a $Q f$ product of $2.6 \times 10^{13} \mathrm{~Hz}$, and the measured value of $Q f=2.7 \times 10^{13} \mathrm{~Hz}$ at $14 \mathrm{MHz}$ at ambient temperatures is slightly higher than this (below the fit curve). These values are close to the highest value of $2.9 \times 10^{13} \mathrm{~Hz}$ previously reported for $Q f$ of LGT by Smythe et al. ${ }^{42}$ They suggest that relatively little additional improvement in room-temperature $Q$ of LGT is possible through material optimization. However, a great deal stands to be gained in resonator performance at temperatures above $600 \mathrm{~K}$, where we find the phonon-phonon loss to be a small fraction of the measured $Q^{-1}$.
The situation with respect to the intrinsic loss in LGS is less clear because of greater uncertainty in $B$ that is introduced by the presence of a larger frequency- and temperature- independent term in the fit $(C)$. In particular, if the physical source of the constant term $C$ is energy loss to the supporting structure through inefficient vibrational trapping, this is expected to introduce an additional frequencydependent component that will confound accurate extraction of the phonon-phonon term $B$. The $Q^{-1}$ arising from inefficient trapping usually decreases with increasing frequency, so that it will partially cancel the phonon-phonon term in Eq. (15). In other words, this effect will decrease the value of $B$ determined from fitting the data and, correspondingly, increase the value determined for the intrinsic $Q f$. The value obtained for $B$ of LGS corresponds to a $Q f$ of $6.4 \times 10^{13} \mathrm{~Hz}$, which is more than a factor of two greater than that obtained here for LGT and five times greater than the highest reported experimental value of $1.3 \times 10^{13} \mathrm{~Hz}$ for LGS at room temperature. $^{42}$

\section{B. Debye relaxations}

The lowest-temperature Debye relaxation in LGS, with an activation energy of $0.24 \mathrm{eV}$ (Eq. (18)), is similar to a previously reported peak in another LGT specimen with an activation energy of $0.25 \mathrm{eV} \pm 0.03 \mathrm{eV} .{ }^{10}$ The second-lowesttemperature peak that we observe in LGS and the corresponding peak in LGT are similar to a peak reported by Fritze et al. ${ }^{2,27}$ to have a maximum between $520 \mathrm{~K}$ and $535 \mathrm{~K}$ at $5 \mathrm{MHz}$ in undoped LGS. Our parameters $U_{2}$ and $\gamma_{2}$ for this peak in LGS correspond to a maximum at $550 \mathrm{~K}$ at $5 \mathrm{MHz}$. Fritze et $a l^{27}$ found no such peak in Sr-doped LGS. Their result is attributable to elimination of the anelastic defect responsible for the peak, and this could involve a change in defect symmetry and associated anelastic properties arising from a dopant-induced change in charge state.

The highest-temperature Debye relaxations are found from our least-squares analysis to have activation energies $U_{3}$ and pre-exponential factors $\gamma_{3}$ that are similar in LGS and LGT. From these fit parameters, the maxima of the peaks are predicted to occur near $883 \mathrm{~K}$ in LGS and $842 \mathrm{~K}$ in LGT at $10 \mathrm{MHz}$. These peaks apparently correspond to a previously reported peak in an LGT cylinder, with a maximum near 817 $\mathrm{K}$ at $1.8 \mathrm{MHz}$, although the activation energies determined from our current analysis are lower than the value of $1.1 \mathrm{eV}$ determined in that earlier study. ${ }^{12}$ The peak temperature for LGS is also close to that reported by Fritze and coworkers ${ }^{2}$ for a damping peak in LGS. In an earlier study over a lower temperature range, Fritze et al. ${ }^{27}$ found the effective LGS viscosity to have an activation energy of $0.88 \mathrm{eV}$ at the highest measured temperatures $\left(\sim 600{ }^{\circ} \mathrm{C}\right.$ to $\left.700{ }^{\circ} \mathrm{C}\right)$, consistent with the value of $0.87 \mathrm{eV}$ that we find for $U_{3}$ of LGS, and they noted the striking similarity of this activation energy to that of $\sigma T$.

\section{Temperature-dependent background in LGS}

Returning to the question of the source of the temperature-dependent background damping in LGS, we note that components of $Q^{-1}$ that increase approximately 
exponentially with $1 / T$ (commonly called the "hightemperature background") have been reported for a wide range of materials. Such damping is not generally well understood and may arise from different physical mechanisms in different materials. Measurements of frequency dependence, which would aid in the evaluation of proposed models, are often not available.

The high-temperature ultrasonic background in quartz has been suggested by Martin ${ }^{43}$ and Martin and Lopez ${ }^{44}$ to arise from alkali ions ( $\mathrm{Li}$ and/or Na) that diffuse through channels along the trigonal axis after they escape from $\mathrm{Al}$ substitutional atoms through thermal excitation. In this model, the relaxation strength is proportional to the concentration of these diffusing anelastic point defects, which, under the assumption of single types of initial trap atoms (Al) and alkali atoms, is proportional to $\exp (-V / k T)$, where $V$ is half the energy of binding of the alkali atom to the trap. ${ }^{44}$ Fraser ${ }^{45}$ found that the high-temperature background in quartz is approximately proportional to $1 / \omega$, which is consistent with the hypothesis of Martin et al ${ }^{43,44}$ that this is the low-temperature shoulder of an anelastic relaxation (Debye function in the high-frequency limit). Since, in our measurements of LGS, the frequency dependence of the temperaturedependent background is found to be much weaker than that of quartz (as reflected in the value of $n$ in Eq. (14)), a model analogous to that of Martin et al. cannot be applied to LGS without substantial modification.

The high-temperature background in pure and alloyed metals has been proposed by a number of authors as arising from anelastic or viscoelastic dislocation motion, and this view is strongly supported by the observed dependence of the magnitude of this background on plastic deformation. For example, Atodirescei, Gremaud, and Schaller ${ }^{46}$ interpreted damping in Al-Mg alloys in terms of an anelastic (single Debye) model, with the distance between strong dislocation pinning points (and associated relaxation strength) dependent on temperature. Weak frequency dependence, when it occurs, also points toward a dislocation mechanism, because variations in dislocation orientations and pinning lengths lead to variations in relaxation rates of dislocation motion under periodic acoustic stress. For example, Weller, Clemens, and Haneczok ${ }^{47}$ interpreted lowfrequency internal friction of intermetallic alloys at high temperatures in terms of a viscoelastic model, with weak frequency dependence formally introduced through fractional derivatives in the equation of motion. ${ }^{48}$ Almost all experimental studies of the high-temperature background in metals have employed frequencies in the range of single-digit hertz to kilohertz (orders of magnitude lower than the ultrasonic frequencies employed in this work), and this makes any application of theoretical models from these studies to our results on LGS challenging. The large differences in dislocation Peierls' barriers between metals and ionic materials also introduce additional questions about the validity of translating these models to LGS.

Studies of the high-temperature background in covalent or ionic single crystals have focused primarily on $\mathrm{Si}, \mathrm{Ge}$, and InSb. ${ }^{49-56}$ With respect to the magnitude of the Peierls' barriers, these studies could offer the most relevant references for considering physical models for LGS. However, the frequencies employed in these studies are, again, far less than those of our LGS measurements. All studies of $\mathrm{Si}$ and $\mathrm{Ge}$ have attributed the high-temperature background to dislocation mechanisms, based largely on the fact that this damping is found to increase with plastic deformation and associated dislocation density. ${ }^{49-52,54}$ However, no consensus of opinion has emerged with respect to the detailed physical mechanism.

The frequency dependence of the high-temperature background in $\mathrm{Si}, \mathrm{Ge}$, and $\mathrm{InSb}$ has been found to be close to $1 / \omega$ at frequencies below $100 \mathrm{kHz}^{50-53,55}$ Therefore, although the reported values of high-temperature $Q^{-1}$ in these materials are, in some cases, comparable in magnitude to the temperature-dependent background found here for LGS, the extrapolation of previous models into the megahertz range leads to values of $Q^{-1}$ far below those found in our measurements on LGS. The reported inverse frequency dependence at low frequencies also is, again, much different from that found here for LGS at ultrasonic frequencies.

We suggest that the high-temperature background in LGS in our measured range of $2 \mathrm{MHz}$ to $14 \mathrm{MHz}$ arises from a physical mechanism different from that which is dominant in low-frequency measurements of $\mathrm{Si}, \mathrm{Ge}$, and InSb. However, the weak frequency dependence in LGS strongly suggests that this background is still associated with dislocations, since distributions in activation energy and attempt frequency will naturally arise from variations in dislocation orientation. A dislocation mechanism is also consistent with the absence of a significant high-temperature background (below $750 \mathrm{~K}$ ) in the fit to $Q^{-1}$ of our LGT specimen, considering that this specimen was found to have a dislocation etch-pit density much less than the LGS specimen.

We hypothesize that the high-temperature background in LGS arises from anelastic motion of individual kinks, rather than the collective motion of entire dislocation lines that is considered in lower frequency studies of other materials. In relation to this hypothesis, the results of theoretical studies of dislocation motion in materials with high Peierls' barriers should be noted. In particular, the extensive theoretical work on Si provides insight into the general form and dynamics of kink mechanisms that can occur, despite the fact that $\mathrm{Si}$ is covalent and has a different crystal symmetry than LGS. To enable an order-of-magnitude comparison of relaxation parameters of kinks in $\mathrm{Si}$ and the fit parameters of the high-temperature background in LGS (Eq. (18)), recent work of Jin, Ren, and Xiang ${ }^{57}$ is particularly useful to note, because it includes calculations of the pre-exponential rate factor, in addition to the activation energy, for kink migration. The activation energies of $0.74 \mathrm{eV}$ to $0.83 \mathrm{eV}$ obtained by Jin, Ren, and Xiang for kink migration on $30^{\circ}$ partial dislocations in $\mathrm{Si}$ (Table I in that work) are close to our value of $0.73 \mathrm{eV}$ for $U_{b}$ in LGS, and the values they obtain for the pre-exponential rate factor are within an order of magnitude of our value for $1 / \gamma_{b}$, lending credibility to our hypothesis of an individual-kink relaxation process. With respect to the possibility of a broad distribution of activation energies for kink migration in LGS (as assumed in the fitting function of 
Eq. (15)), we note that there are multiple kink migration pathways in $\mathrm{Si}$, and some of these are predicted to have very low activation energies. Recent theoretical calculations of screw dislocations in Si have yielded activation energies in the range of $0.07 \mathrm{eV}$ to $0.18 \mathrm{eV} .^{58,59}$ Soliton mediation has also been considered as a possible mechanism for greatly lowering activation energies of $90^{\circ}$ partial (pure edge) dislocations in $\mathrm{Si}^{60,61}$

The simple log-normal distribution of activation energies in the background term in Eq. (18) seems, however, unlikely to be a highly accurate approximation of the actual distribution of relaxations in LGS. This specific continuous function is employed here for the sake of simplicity and robustness of the fit. The possibilities of a distribution of $\gamma_{b}$ and temperature dependence of $\Delta_{b}$ in the function $Q_{2}^{-1}$ also are not considered. With respect to the hypothesized kinkmigration mechanism, we note that thermal generation of kinks would be expected to introduce a contribution to $\Delta_{b}$ with an Arrhenius form.

Theories and measurements of low-temperature relaxations in metals that are attributed to kinks should also be noted. The Bordoni peak, which occurs at cryogenic temperatures in low-frequency internal friction measurements of metals, is generally interpreted in terms of kink pair formation, rather than migration of kinks that are thermally nucleated or required by dislocation orientation (geometric). ${ }^{62}$ In many theoretical approaches, the migration of kinks is assumed to be so rapid that the corresponding timescales are insignificant in low-frequency measurements of the Bordoni relaxation. However, Marchesoni ${ }^{63}$ has presented a theoretical model in which the "background" attenuation that is found to increase with increasing temperature in some low-frequency measurements of the Bordoni peak(s) arises from kink migration. This contribution in his model is dependent on the pinning length of the dislocations. In a sample calculation for copper, Marchesoni found a monotonically increasing background over the calculated temperature range (extending to approximately twice the temperature of the Bordoni peak), assuming a Koehler ${ }^{40}$ distribution of dislocation lengths and a distribution of kink densities. The potential applicability of this model to the interpretation of high-temperature ultrasonic damping in LGS and other piezoelectric materials might be useful to explore in future work.

\section{LGS versus LGT}

The measurements and analysis presented above reveal higher defect-related contributions to $Q^{-1}$ in our LGS specimen than in our LGT specimen over a broad range of temperatures. Because of differences in the growth of these two crystals, including oxygen content of the growth atmosphere, this result does not provide a direct comparison of the relative limitations on $Q$ in LGS and LGT. However, our result is qualitatively in line with previously reported results of Smythe et al., ${ }^{42}$ showing generally higher $Q^{-1}$ in LGS than in LGT at ambient temperatures. Although it remains to be determined whether these conclusions about relative magnitudes of $Q^{-1}$ in LGS and LGT are broadly applicable, we see fundamental differences in the crystal growth processes of
LGS and LGT that present greater obstacles to the production of LGS with low densities of defects, including dislocations. LGS is grown from a melt containing silicon oxide and, therefore, having relatively high viscosity. In such melts, diffusion is inefficient, so that the creation of a homogeneous liquid is challenging. Common effects of this situation are the presence of inclusions in LGS crystals and associated generation of dislocations (arising from thermal stresses) during cooling. One approach for dealing with this issue of melt homogenization is to overheat the melt to bring the oxides into solution. A second approach is to presynthesize the starting oxides and then melt the solid-state sintered LGS. A third approach is to increase the soaking time of the melt prior to growth. A combination of such approaches is also possible. Overheating and longer soaking times result in enhanced (preferential) and time-dependent evaporation of gallium oxide. Hence, the stoichiometry of the melt will change with time. Overheating also results in the formation of secondary phases that will be incorporated in the crystal as inclusions. Therefore, optimizing the heating of LGS during crystal growth is challenging. In contrast, since LGT growth does not involve silicon oxide, melts are not overly viscous and homogeneity is more easily achieved.

Another issue in the growth of LGS and LGT is the formation of facets at the liquid/solid interface. The presence of several different orientations of facets leads to inhomogeneities in crystals and associated internal stress and dislocation generation during cooling. ${ }^{64}$ Low-index facets develop when the orientation of the freezing-point isotherm is close to the orientation of a crystallographic facet. This is a wellknown issue for other oxide crystals, for example, yttriumaluminum-garnet (YAG) laser-host crystals. The two common approaches to solving this problem are to grow the crystals in an orientation that will not lead to facet formation and to adjust the shape of the liquid/solid interface such that it does not fall into a faceting plane. The problem of faceting in LGS has not yet been solved. In the case of LGT, Klemenz et al. ${ }^{64}$ demonstrated crystals to be free of (low-index) facets after optimization of the crystal growth process. The LGT resonator used in this study came from such a crystal.

As we have previously suggested, ${ }^{11}$ LGT also appears to offer another advantage, relative to LGS, in minimizing the contribution of the lowest-temperature point-defect relaxation to $Q^{-1}$ at ambient temperatures, even if the concentrations of the defects responsible for this relaxation are comparable in the two materials. This relaxation has been found to occur at lower cryogenic temperatures in LGT, so that its contribution to $Q^{-1}$ is less at ambient temperatures. ${ }^{11}$

\section{CONCLUSION}

Measurements and analysis of temperature-dependent $Q^{-1}$ of LGS and LGT reveal a superposition of several physical effects, including point-defect relaxations and intrinsic phonon-phonon loss. The LGS specimen measured here also has a large temperature-dependent background arising from a distributed relaxation process, which we have suggested is consistent with anelastic kink migration along dislocations in response to ultrasonic stress. The piezoelectric/carrier 
relaxation is found not to be a significant contribution to $Q^{-1}$ in either LGS or LGT over the measured range of temperatures. Interpretation of the data at the highest measured temperatures is made more challenging by the fact that a dominant point-defect relaxation at these temperatures has an activation energy close to that expected for the higher temperature piezoelectric/carrier relaxation.

One conclusion of this work is that $Q^{-1}$ of our LGT specimen at ambient temperatures is already dominated by the intrinsic phonon-phonon loss, so that there is little room for additional improvement in $Q^{-1}$ at these temperatures. This conclusion is consistent with the best reported $Q f$ of LGT at ambient temperature being only $7 \%$ greater than our value measured at $14 \mathrm{MHz}^{42}$

At higher temperatures in the measured range, $Q^{-1}$ is dominated by defect-related relaxations. These sources of damping can be reduced or eliminated, at least in LGT. This is illustrated by the smaller defect-related contributions to $Q^{-1}$ of the present LGT specimen, relative to a previously measured LGT specimen ${ }^{12}$ and the present LGS specimen. With respect to reducing the second relaxation in LGS (586 $\mathrm{K}$ at $10 \mathrm{MHz}$ ) and the corresponding relaxation in LGT (504 $\mathrm{K}$ at $10 \mathrm{MHz}$ ), one should note the work of Fritze et al., ${ }^{27}$ which shows the peak in LGS to be essentially eliminated by Sr doping.

At temperatures well above the measured range, the lower limit of $Q^{-1}$ is ultimately determined by the piezoelectric/ carrier relaxation. As described in Sec. V, the relaxation strength $U_{c}$ is determined entirely by the elastic, piezoelectric, and dielectric constants, which are intrinsic properties for a given crystal orientation and acoustic mode. Therefore, the only way to substantially reduce the magnitude of the piezoelectric/carrier damping at a given temperature is to shift this relaxation peak to higher temperatures. This can be accomplished by either reducing the electrical conductivity through appropriate doping or increasing the operating frequency of the resonator. At temperatures well below the peak maximum, the Debye function for the piezoelectric/carrier contribution to $Q^{-1}$ (Eq. (8)) is in the high-frequency regime and approximately proportional to $\sigma / \omega$.

Over much of the measured temperature range, the temperature-dependent background is the greatest contribution to $Q^{-1}$ in the LGS specimen studied here. This background presents a challenge to physical interpretation, due to the form of its combined dependence on temperature and frequency. No evidence has been found for a significant background of this form in the LGT specimen, which had lower dislocation etch-pit density, consistent with our hypothesis that this contribution to $Q^{-1}$ in LGS is associated with dislocations. However, our hypothesis of a kink mechanism is highly tentative, and, considering the limited conclusions of past research on high-temperature damping in other materials, it may be anticipated to require substantial research to verify or disprove. In any case, the fact that we have found no evidence for such a background in our LGT specimen, over the measured temperature range, is encouraging, with respect to the possibility that this contribution to $Q^{-1}$ will not present a substantial obstacle to higher temperature operation of state-of-the-art LGT resonators.

\section{ACKNOWLEDGMENTS}

We are grateful to Mitsubishi Materials Corporation for supplying and characterizing the LGS crystal. We also appreciate the contribution of employees of MTronPTI, including Eric Hague and Robert Smythe, in fabricating and characterizing the resonators, and we appreciate the contribution of Damian Lauria in developing computer programs to control the ultrasonic experiments.

${ }^{1}$ B. V. Mill and Y. V. Pisarevsky, in Proceedings of the 2000 IEEE/IEA International Frequency Control Symposium (IEEE, Piscataway, 2000), p. 133.

${ }^{2}$ H. Fritze, Meas. Sci. Technol. 22, 012002 (2011).

${ }^{3}$ J. A. Kosinski, R. A. Pastore, Jr., E. Bigler, M. P. da Cunha, D. C. Malocha, and J. Detaint, in Proceedings of the 2001 IEEE International Frequency Control Symposium and PDA Exhibition (IEEE, Piscataway, 2001), p. 278.

${ }^{4}$ B. T. Sturtevant, P. M. Davulis, and M. P. da Cunha, IEEE Trans. Ultrason. Ferroelectr. Freq. Control 56, 788 (2009).

${ }^{5}$ J. J. Boy, R. J. Besson, E. Bigler, R. Bourquin, and B. Dulmet, in Proceedings of the 2001 IEEE International Frequency Control Symposium (IEEE, Piscataway, 2001), p. 223.

${ }^{6}$ Y. Kim and A. Ballato, IEEE Trans. Ultrason. Ferroelectr. Freq. Control 50, 1678 (2003).

${ }^{7}$ Y. Jing, J. Chen, X. Gong, and J. Duan, IEEE Trans. Ultrason. Ferroelectr. Freq. Control 54, 906 (2007).

${ }^{8}$ J. A. Kosinski, R. A. Pastore, J. Yang, and J. A. Turner, IEEE Trans. Ultrason. Ferroelectr. Freq. Control 56, 129 (2009).

${ }^{9}$ H. Fritze, J. Electroceram. 17, 625 (2006).

${ }^{10}$ W. L. Johnson, S. A. Kim, D. S. Lauria, and R. C. Smythe, in Proceedings of the 2002 IEEE Ultrasonics Symposium (IEEE, Piscataway, 2002), p. 961.

${ }^{11}$ W. L. Johnson, S. A. Kim, and S. Uda, in Proceedings of the 2003 IEEE International Frequency Control Symposium (IEEE, Piscataway, 2003), p. 646.

${ }^{12}$ W. Johnson, S. Kim, and D. Lauria, in Proceedings of the 2000 IEEE/EIA International Frequency Control Symposium (IEEE, Piscataway, 2000), p. 186.

${ }^{13}$ M. Schulz, J. Sauerwald, D. Richter, and H. Fritze, Ionics 15, 157 (2009).

${ }^{14}$ J. Schreuer, J. Rupp, C. Thybaut, and J. Stade, in Proceedings of the 2002 IEEE Ultrasonics Symposium (IEEE, Piscataway, 2002), p. 373.

${ }^{15}$ J. Schreuer, C. Thybaut, M. Prestat, J. Stade, and E. Haussühl, in Proceedings of the 2003 IEEE Ultrasonics Symposium (IEEE, Piscataway, 2003), p. 196.

${ }^{16}$ R. C. Smythe, in Proceedings of the 1998 IEEE International Frequency Control Symposium (IEEE, Piscataway, 1998), p. 761.

${ }^{17}$ G. M. Kuzmicheva, E. N. Domoroshina, V. B. Rybakov, A. B. Dubovsky, and E. A. Tyunina, J. Cryst. Growth 275, e715 (2005).

${ }^{18}$ G. M. Kuz'micheva, I. A. Kaurova, V. B. Rybakov, A. B. Dubovsky, A. Cousson, and O. Zaharko, Crystallogr. Rep. 55, 1067 (2010).

${ }^{19}$ N. Bamba, K. Kato, T. Taishi, T. Hayashi, K. Hoshikawa, and T. Fukami, Mater. Sci. Forum 510-511, 842 (2006).

${ }^{20}$ H. Kimura, S. Uda, O. Buzanov, X. Huang, and S. Koh, J. Electroceram. 20, 73 (2008).

${ }^{21}$ I. A. Kaurova, G. M. Kuz'micheva, V. B. Rybakov, A. B. Dubovskii, and A. Cousson, Inorg. Mater. 46, 988 (2010).

${ }^{22}$ W. Johnson, J. Phys. IV 6(C8), 849 (1996).

${ }^{23}$ W. Johnson, J. Appl. Phys. 83, 2462 (1998).

${ }^{24}$ E. P. EerNisse, in Proceedings of the 2000 IEEE/EIA International Frequency Control Symposium (IEEE, Piscataway, 2000), p. 331.

${ }^{25}$ J. Bardong, M. Schulz, M. Schmitt, I. Shrena, D. Eisele, E. Mayer, L. M. Reindl, and H. Fritze, in Proceedings of the 2008 IEEE International Frequency Control Symposium (IEEE, Piscataway, 2008), p. 326.

${ }^{26}$ H. Fritze, O. Schneider, H. Seh, H. L. Tuller, and G. Borchardt, Phys. Chem. Chem. Phys. 5, 5207 (2003).

${ }^{27}$ H. Fritze, M. Schulz, H. Seh, H. L. Tuller, S. Ganschow, and K. Jacobs, Solid State Ionics 177, 3171 (2006).

${ }^{28}$ A. Akhieser, J. Phys. (USSR) 1, 277 (1939).

${ }^{29}$ W. P. Mason, in Physical Acoustics, Principles and Methods, edited by W. . Mason (Academic, New York, 1965), Vol. 3B. 
${ }^{30}$ A. S. Nowick and B. S. Berry, Anelastic Relaxation in Crystalline Solids (Academic, New York, 1972).

${ }^{31}$ A. R. Hutson and D. L. White, J. Appl. Phys. 33, 40 (1962).

${ }^{32}$ W. P. Mason, in Physical Acoustics, Principles and Methods, edited by W. P. Mason (Academic, New York, 1964), Vol. 1A.

${ }^{33}$ B. A. Auld, Acoustic Fields and Waves in Solids (Krieger, Malabar, FL, 1990), Vol. I.

${ }^{34}$ H. Seh and H. L. Tuller, J. Electroceram. 16, 115 (2006).

${ }^{35} \mathrm{~J}$. Bohm, R. B. Heimann, M. Hengst, R. Roewer, and J. Schindler, J. Cryst. Growth 204, 128 (1999).

${ }^{36}$ E. N. Domoroshchina, A. B. Dubovskii, G. M. Kuz'micheva, and G. V. Semenkovich, Inorg. Mater. 41, 1218 (2005).

${ }^{37}$ M. Schulz and H. Fritze, Renewable Energy 33, 336 (2008).

${ }^{38} \mathrm{P}$. Davulis and M. P. da Cunha, in Proceedings of the 2010 IEEE Frequency Control Symposium (IEEE, Piscataway, 2010), p. 252.

${ }^{39}$ I. A. Dankov, K. V. Novichkov, E. F. Tokarev, S. A. Sakharov, and O. A. Buzanov, Meas. Tech. 53, 134 (2010).

${ }^{40}$ J. S. Koehler, in Imperfections in Nearly Perfect Crystals, edited by W. Shockley, J. H. Holloman, R. Maurer, and F. Seitz (Wiley, New York, 1952), p. 197.

${ }^{41}$ A. Granato and K. Lücke, J. Appl. Phys. 27, 583 (1956).

${ }^{42}$ R. C. Smythe, R. C. Helmbold, G. E. Hague, and K. A. Snow, IEEE Trans. Ultrason. Ferroelectr. Freq. Control 47, 355 (2000).

${ }^{43}$ J. J. Martin, in Proceedings of the 1996 IEEE International Frequency Control Symposium (IEEE, Piscataway, 1996), p. 170.

${ }^{44}$ J. J. Martin and A. R. Lopez, in Proceedings of the 2001 IEEE International Frequency Control Symposium (IEEE, Piscataway, 2001), p. 316.

${ }^{45}$ D. B. Fraser, J. Appl. Phys. 35, 2913 (1964).

${ }^{46}$ M. Atodirescei, G. Gremaud, and R. Schaller, Mater. Sci. Eng., A 442, 160 (2006).
${ }^{47}$ M. Weller, H. Clemens, and G. Haneczok, Mater. Sci. Eng., A 442, 138 (2006).

${ }^{48}$ G. Haneczok and M. Weller, Mater. Sci. Eng., A 370, 209 (2004).

${ }^{49}$ J. O. Kessler, Phys. Rev. 106, 646 (1957).

${ }^{50}$ P. D. Southgate, Proc. Phys. Soc. London 76, 398 (1960).

${ }^{51}$ P. D. Southgate and A. E. Attard, J. Appl. Phys. 34, 855 (1963).

${ }^{52}$ P. D. Southgate and K. S. Mendelson, J. Appl. Phys. 36, 2685 (1965).

${ }^{53}$ F. Calzecchi, P. Gondi, and S. Mantovani, J. Appl. Phys. 40, 4798 (1969).

${ }^{54}$ G. Welsch, T. E. Mitchell, and R. Gibala, Phys. Status Solidi A 15, 225 (1973).

${ }^{55}$ A. P. Gerk and W. S. Williams, J. Appl. Phys. 53, 3585 (1982).

${ }^{56}$ U. Jendrich and P. Haasen, Phys. Status Solidi A 108, 553 (1988).

${ }^{57}$ C. Jin, W. Ren, and Y. Xiang, Scr. Mater. 62, 206 (2010).

${ }^{58}$ L. Pizzagalli, A. Pedersen, A. Arnaldsson, H. Jónsson, and P. Beauchamp, Phys. Rev. B 77, 064106 (2008).

${ }^{59}$ A. Pedersen, L. Pizzagalli, and H. Jónsson, J. Phys.: Condens. Matter 21, 084210 (2009).

${ }^{60}$ R. W. Nunes, J. Bennetto, and D. Vanderbilt, Phys. Rev. Lett. 77, 1516 (1996).

${ }^{61}$ S. Scarle, C. P. Ewels, M. I. Heggie, and N. Martsinovich, Phys. Rev. B 69, 075209 (2004)

${ }^{62}$ G. Schoeck, Philos. Mag. 86, 3819 (2010).

${ }^{63}$ F. Marchesoni, Phys. Rev. Lett. 74, 2973 (1995).

${ }^{64}$ C. F. Klemenz, J. Luo, and D. Shah, in Advances in Electronic Ceramic Materials, Ceramic Engineering and Science Proceedings 26 (American Ceramic Society, Westerville, 2005), p. 169.

${ }^{65}$ The identification of the manufacturer of the LGS crystal is provided here for technical completeness and does not reflect an endorsement by NIST. The properties that we measure for this LGS crystal, which was supplied in 2002, are also not intended to be representative of LGS currently manufactured by Mitsubishi. 